\title{
Mining the XRT archive to probe the X-ray absorber structure in the AGN population
}

\author{
Lucia Ballo* \\ Osservatorio Astronomico di Brera (INAF), via Brera 28, I-20121, Milano (Italy); E-mail: \\ lucia.ballo@brera.inaf.it \\ Paola Severgnini, Alberto Moretti, Roberto Della Ceca, Stefano Andreon, \\ Alessandro Caccianiga \\ Osservatorio Astronomico di Brera (INAF), via Brera 28, I-20121, Milano (Italy)

\section{Valentina Braito} \\ Osservatorio Astronomico di Brera (INAF), via E. Bianchi 46, I-23807 Merate, LC (Italy); \\ Department of Physics, University of Maryland, Baltimore County, Baltimore, MD 21250 (USA)
}

\section{Sergio Campana}

Osservatorio Astronomico di Brera (INAF), via E. Bianchi 46, I-23807 Merate, LC (Italy)

\section{Cristian Vignali}

Dipartimento di Fisica e Astronomia, Università degli Studi di Bologna, viale Berti Pichat 6/2,

I-40127, Bologna (Italy);

Osservatorio Astronomico di Bologna (INAF), via Ranzani 1, I-40127, Bologna (Italy)

\begin{abstract}
One of the key ingredients of the Unified Model of Active Galactic Nuclei (AGN) is the presence of a torus-like optically thick medium composed by dust and gas around the putative supermassive black hole. However, the structure, size and composition of this circumnuclear medium are still matter of debate. To this end, the search for column density variations through X-ray monitoring on different timescales (months, weeks and few days) is fundamental to constrain size, kinematics and location of the X-ray absorber(s).

Here we describe our project of mining the Swift-XRT archive to assemble a sample of AGN with extreme column density variability and determining the physical properties of the X-ray absorber(s). We also present the results obtained from a daily-weekly Swift-XRT follow-up monitoring recently performed on one of the most interesting new candidates for variability discovered so far, Mrk 915.
\end{abstract}

Swift: 10 Years of Discovery,

2-5 December 2014

La Sapienza University, Rome, Italy

${ }^{*}$ Speaker. 


\section{Variable $S$ wift-AGN sample}

According to the Unified Model of Active Galactic Nuclei (AGN; [1]), an obscuring optically thick medium composed by dust and gas arranged in a torus-like geometry is present around the nuclear engine. However, the structure, size and composition of this circumnuclear medium are still matter of debate; important constrains have been recently provided by the study of the absorption variability, almost ubiquitous in bright absorbed AGN ([2]). X-ray absorbing column densities, $N_{\mathrm{H}}$, have been observed to change over different timescales, from a few years down to weeks or days, for a handful of AGN (e.g., [3] and references therein), allowing us to investigate the X-ray properties of the absorbers down to sub-parsec scales. The emerging picture is that multiple neutral and ionized absorbers co-exist around the central black hole, located at different distances from it.

We recently started a project aimed at finding more examples of these sources in order to study the physical properties of the X-ray absorbers on a larger statistical basis. We exploited the vaste amount of X-ray observations provided by the X-ray Telescope (XRT; [4]) onboard the satellite Swift. XRT long exposure observations are always split in segments of $\sim 1 \mathrm{ks}$, with typical integration times of $5 \mathrm{ks}$ per day. This generates, for bright sources, a natural short-timescale monitoring, with spectra good enough to perform a basic spectral analysis. As an example, for an AGN with a flux of $F_{0.5-10 \mathrm{keV}} \sim 5 \times 10^{-12} \mathrm{ergs} \mathrm{cm}^{-2} \mathrm{~s}^{-1}$, in $\sim 5 \mathrm{ks}$ we expect to collect $\sim 500$ net photons. This makes the Swift-XRT archive an important database to search for different timescale variability of bright sources. To assemble our sample, we considered:

1. the serendipitous sources detected in the field of view of XRT observations performed as follow-up of 76 gamma-ray bursts. These observations correspond to $\sim 49$ square degrees;

2. the XRT sources classified as AGN that are possible counterparts of objects detected by the Burst Alert Telescope (BAT) onboard Swift [5, 6]. This AGN sample can be considered unbiased in term of column density, at least for $N_{\mathrm{H}} \lesssim 10^{24} \mathrm{~cm}^{-2}$ (a limit above which a source falls in the so-called Compton-thick regime). In the energy band covered by BAT $(14-195 \mathrm{keV})$, the intrinsic emission is indeed weakly affected by absorption levels below few $10^{24} \mathrm{~cm}^{-2}$.

For each source, we split the XRT data sets on a daily basis, combining all the observation segments within 1 day (for the AGN-BAT sample) or 2 days (for the serendipitous sources). We filtered out time bins shorter than $200 \mathrm{sec}$. The light curves span a wide range of number of time bins (from one up to more than 200 spectra) and timescales (from few days to years). For 299 serendipitous sources and 405 BAT AGN we have at least two spectra.

As a proxy of the amount of column density we used the hardness ratio $H R=(H-S) /(H+S)$, where $S=2-4 \mathrm{keV}$ and $H=4-10 \mathrm{keV}$. In the $2-10 \mathrm{keV}$ energy band, the spectrum of an AGN can be approximate, at the first order, by an absorbed power law, while below $\sim 2 \mathrm{keV}$ different soft components (soft excess, reflection, scattering, etc) are also present. Under this hypothesis, the $H R$ value as defined here provides a strong indication of the amount of absorption: in Fig. 1 (left panel) we compare the $H R$ vs. $N_{\mathrm{H}}$ derived from the analysis of the XRT data with the $H R$ measured for a number of simulated spectra (photon index $\Gamma=2$ ) with different values of absorbing column density. For each input $N_{\mathrm{H}}$ value, we indicate with different colours the $16^{\text {th }}$ and $84^{\text {th }}$ percentile of the distribution of 100 tests with three different values of $2-10 \mathrm{keV}$ source photons (from lightest to darkest, 20, 100 and 500). From the plot it is evident that the $H R$ is a proxy of the $N_{\mathrm{H}}$, and 

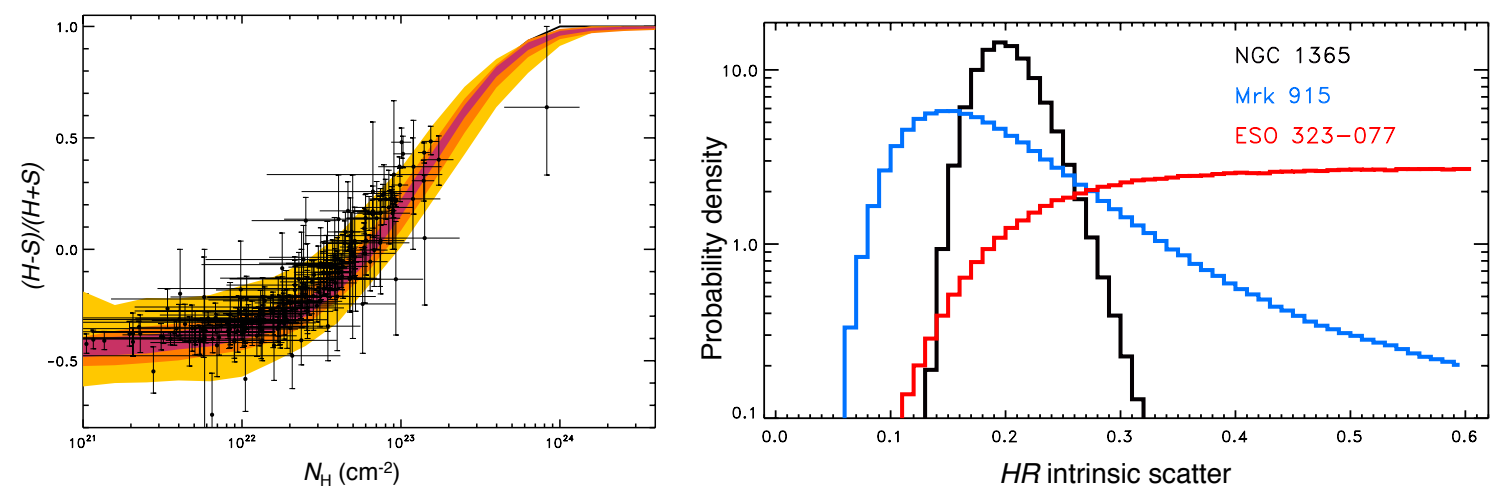

Figure 1: Left panel: $H R$ of simulated spectra $(\Gamma=2)$ for different values of $N_{\mathrm{H}}$. Shaded bands: $16^{\text {th }}$ and $84^{\text {th }}$ percentile from 100 simulations assuming source counts from 20 (yellow) to 500 (purple). Superimposed, we plot the real values derived by the XRT data for the BAT AGN considered here (black dots and error bars). Right panel: Examples of posterior probability of $H R$ intrinsic scatter for 3 sources for which spectral variability has been observed at $99.99 \ldots \%$ confidence level.
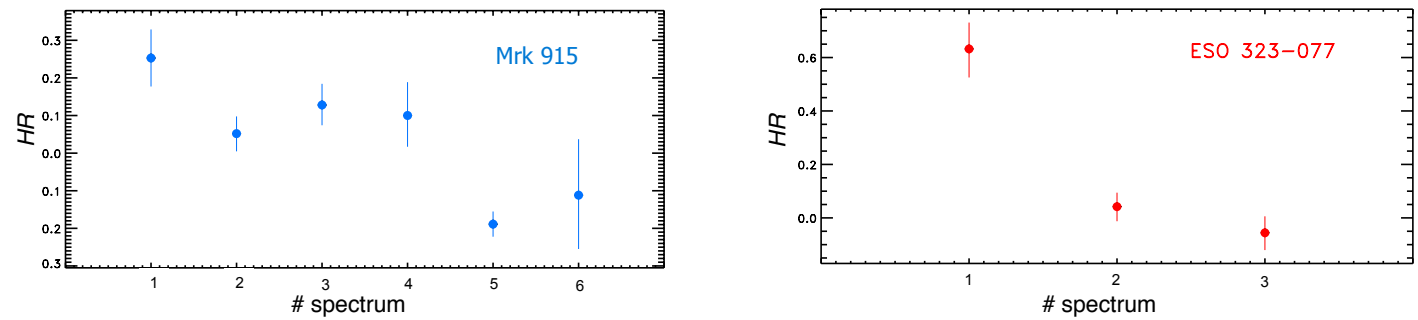

Figure 2: Observed $H R$ light curves derived from the XRT archival data binned on a daily basis for two of the variable candidates (Mrk 915, left; and ESO 323-077, right).

variation in the $H R$ can be interpreted as an indication of a change in the absorbing column density, at least for $N_{\mathrm{H}}$ between $\sim 10^{22} \mathrm{~cm}^{-2}$ and few times $10^{23} \mathrm{~cm}^{-2}$.

In order to probe the spectral variability, for each source we measured the possible non-zero amplitude of an intrinsic $H R$ scatter superimposed to the statistical one. We adopted a Bayesian approach similar to the one used by [7] to determine the intrinsic scatter of cluster velocity dispersion in presence of measurement errors, adapted here to Poisson-based quantities. Basically, starting from the source and background photons in the two bands $(2-4 \mathrm{keV}$ and $4-10 \mathrm{keV})$ and in the different time bins, we calculate the probability for each value of intrinsic scatter given the data. As an example, the probability distributions for 3 sources for which a spectral variation is clearly detected are shown in Fig. 1 (right panel), where we plot the probability associated to an intrinsic scatter value ranging from 0 (corresponding to the no-scatter case) to 0.6. As evident from the figure, the probability of null intrinsic scatter is $\ll 10^{-3}$, implying a negligible probability that the measured $H R$ variability (shown in Fig. 2 for two of the sources) is due to statistical fluctuations.

By applying a very conservative threshold of $10^{-3}$ in the probability of null intrinsic scatter, we found 15 AGN candidates for variable absorbers, 2 of which are serendipitous and 13 are BAT-selected. The sources are listed in Table 1, together with the main characteristics of the XRT archival data considered here. For the AGN observed by BAT, we summarise also the basic information extracted from the Swift-BAT 70 month survey catalogue ${ }^{1}$. Most of the BAT-selected sources are well-known variable AGN: this confirms the validity of the adopted method. We are

\footnotetext{
${ }^{1}$ See http://swift.gsfc.nasa.gov/results/bs70mon/
} 


\begin{tabular}{|c|c|c|c|c|c|c|c|c|}
\hline \multirow{2}{*}{$\begin{array}{c}\text { Source name } \\
\text { (1) }\end{array}$} & \multicolumn{3}{|c|}{ XRT archive } & \multicolumn{4}{|c|}{ BAT catalogue } & \multirow{2}{*}{$\begin{array}{l}\text { Ref. } \\
\text { (9) }\end{array}$} \\
\hline & $\begin{array}{l}\text { Tot. Exp. } \\
\text { (2) }\end{array}$ & $\begin{array}{c}\langle\text { count rate }\rangle \\
\text { (3) }\end{array}$ & $\begin{array}{c}\text { \# spectra } \\
\text { (4) }\end{array}$ & $\begin{array}{c}F_{14-195 \mathrm{keV}} \\
(5)\end{array}$ & $\begin{array}{c}\Gamma_{14-195 \mathrm{keV}} \\
(6)\end{array}$ & $\begin{array}{c}z \\
(7)\end{array}$ & $\begin{array}{l}\text { Opt. class. } \\
\text { (8) }\end{array}$ & \\
\hline Mrk 915 & 23.2 & 0.14 & 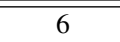 & $31 \pm 6$ & 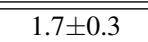 & 0.02411 & $\overline{\text { Sy1 }}$ & \\
\hline NGC 1365 & 154.8 & 0.14 & 34 & $64 \pm 4$ & $2.0 \pm 0.1$ & 0.00546 & Sy 1.8 & {$[8,9,10,11,12]$} \\
\hline ESO $362-18$ & 96.9 & 0.53 & 31 & $49 \pm 4$ & $1.9 \pm 0.1$ & 0.01245 & Sy1.5 & [13] \\
\hline CGCG 031-072 & 10.7 & 0.03 & 3 & $21 \pm 5$ & $1.9 \pm 0.3$ & 0.03305 & Sy1 & \\
\hline NGC 4395 & 243.6 & 0.11 & 190 & $25_{-3}^{+4}$ & $2.0 \pm 0.2$ & 0.00106 & Sy 1.9 & {$[14,12]$} \\
\hline ESO 323-077 & 7.5 & 0.09 & 3 & 33 & 2.1 & 0.01501 & Sy1.2 & {$[15]$} \\
\hline Mrk 335 & 299.2 & 0.34 & 182 & $18 \pm 4$ & $2.3 \pm 0.3$ & 0.02578 & Sy 1.2 & {$[16,17,18,3]$} \\
\hline NGC 3227 & 14.5 & 0.32 & 6 & $10 \pm 4$ & $2.0 \pm 0.1$ & 0.00386 & Sy1.5 & {$[14,3,12]$} \\
\hline NGC 3516 & 36.8 & 0.46 & 31 & $118 \pm 4$ & $1.9 \pm 0.1$ & 0.00884 & Sy1.5 & {$[19,20,3,12]$} \\
\hline NGC 4051 & 39.6 & 0.67 & 32 & $40 \pm 3$ & $2.3 \pm 0.1$ & 0.00233 & Sy1.5 & {$[21,22,14,3,12]$} \\
\hline Mrk 766 & 108.5 & 0.58 & 56 & $22 \pm 3$ & $2.5 \pm 0.2 \pm$ & 0.01293 & Sy 1.5 & {$[23,24,25,26,14]$} \\
\hline NGC 3783 & 21.7 & 2.2 & 6 & $181 \pm 5$ & $2.0 \pm 0.1$ & 0.00973 & Sy1 & {$[27,14,3,12]$} \\
\hline $\mathrm{MCG}+11-11-032$ & 14.6 & 0.02 & 3 & $18 \pm 4$ & $1.8 \pm 0.3$ & 0.036 & Sy2 & \\
\hline SWJ012118-125727 & 53.6 & 0.01 & 4 & & & & & \\
\hline SWJ105826-231414 & 59.4 & 0.004 & 7 & & & & & \\
\hline
\end{tabular}

Table 1: Swift-based information for the 15 objects that our method candidates as variable with high confidence. We list in the first part the 13 BAT-selected AGN, and in the second part the 2 serendipitous sources. Col. (1): Source name. Cols. (2) - (4): Information related with the XRT archival data used in this work: total exposure time, in units of ksec; average $0.5-10 \mathrm{keV}$ count rate; number of useful spectra. Cols. (5) - (8): Information derived from the Swift-BAT 70 month survey catalogue: BAT flux in the $14-195 \mathrm{keV}$ energy band, in units of $10^{-12} \mathrm{ergs} \mathrm{cm}^{-2} \mathrm{~s}^{-1}$; spectral index (computed from a power-law fit to the 8-band BAT data); redshifts (taken from the online databases NED and SIMBAD); optical classification. Col. (9): Not-exhaustive list of previous studies of variability in the X-ray band.

exploring its potentiality by relaxing the threshold in probability of null intrinsic scatter. However, even the more restrictive threshold used here demonstrates the effectiveness of this method: it provided us with 5 new candidates (two of which are serendipitous) that were not previously known as variable sources. Below we present the results obtained from a daily-weekly Swift-XRT followup monitoring that was recently performed on one of them, Mrk 915.

\section{A new candidate for variable absorber: Mrk 915}

Mrk $915(z=0.024)$ is a Seyfert 1.5-1.9 galaxy hosting a black hole of mass $M_{\mathrm{BH}}=0.6-$ $1.8 \times 10^{8} M_{\odot}([28])$. Spectroscopic observations performed in the optical band ([28, 29]) suggest that part of the optical absorption affecting the central regions originates outside the obscuring torus, and it is most likely associated with the dust lanes seen crossing the central source ([30]). For this source the $H R$ variation observed has a probability less than $10^{-6}$ of having a null intrinsic scatter superimposed to the statistical fluctuations (Fig. 2, left panel).

We were awarded 12 observations with XRT (covering $\sim 3$ weeks, for a total of $76 \mathrm{ksec}$ net exposure time), to further investigate the $N_{\mathrm{H}}$ variability and its timescales. In Fig. 3 we show the temporal behaviour of Mrk 915, as reconstructed considering all the Swift datasets (archival on the left panel, new monitoring on the right panel). The data are binned both per observation (red filled circles) and per snapshot (grey dots). Comparing the $0.3-10 \mathrm{keV}$ light curves from the new monitoring and the archival data, we confirm, on shorter timescales, the count rate variability previously detected (see Fig. 3, upper panels). However, the analysis of the ratio of count rates observed in hard and soft bands $(H / S)$ highlights a change in the behaviour of the source: in the archival observations Mrk 915 underwent to a dramatic spectral variation on a timescale of 1 month. This is clearly revealed by the decreasing of the $4-10 \mathrm{keV}$ vs. $0.3-4 \mathrm{keV}$ ratio of a factor of 3 

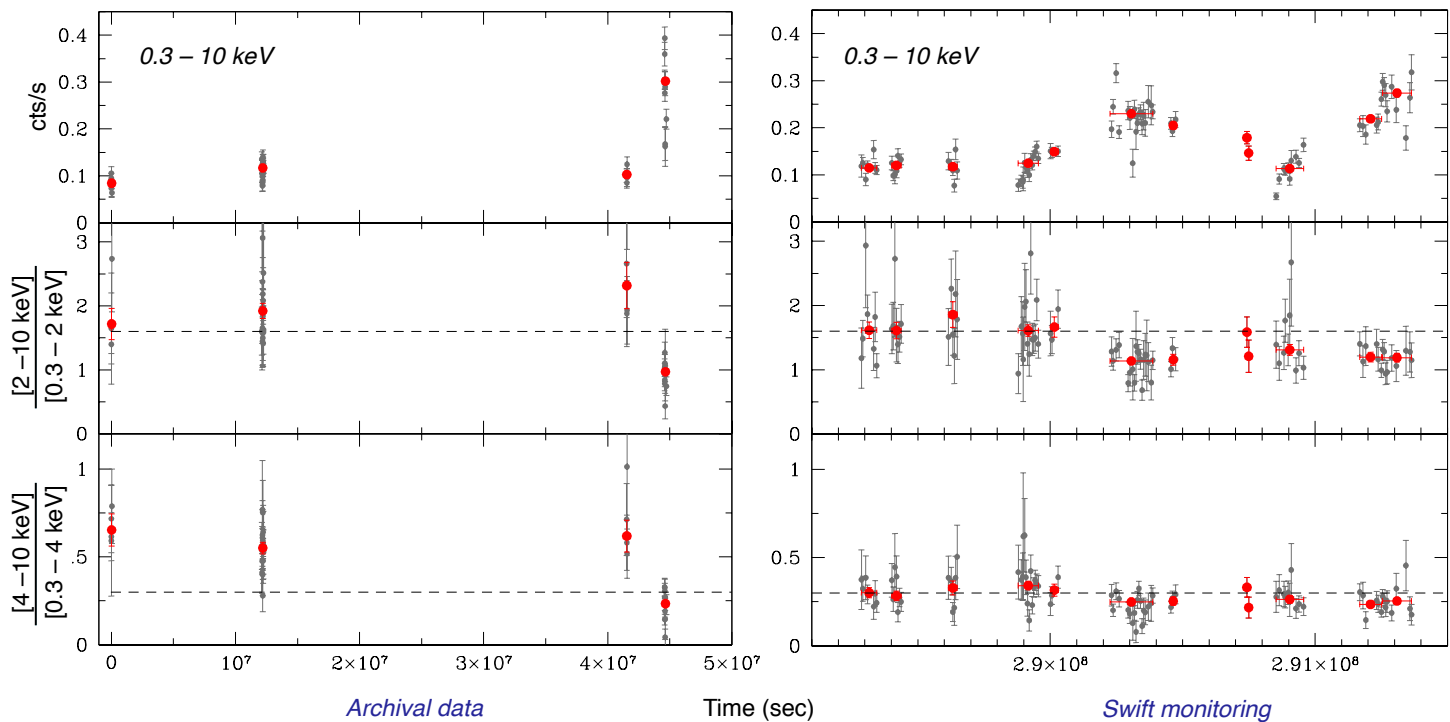

Figure 3: Light curves of the $0.3-10 \mathrm{keV}$ count rate (upper panels) and of the count rate ratios in the energy ranges $2-10 \mathrm{keV}$ vs. $0.3-2 \mathrm{keV}$ (middle panels) and $4-10 \mathrm{keV}$ vs. $0.3-4 \mathrm{keV}$ (lower panels). We compare the trend observed in the archival data of Mrk 915 (left panels) with the results from the September 2014 Swift monitoring (right panels). As time zero-point, the starting time of the first archival observation is assumed. Grey dots: one bin per snapshot; red filled circles: one bin per observation. To highlight the observed variation in the $H / S$, we mark with grey dashed lines the mean ratio observed in the first observation of the monitoring. From Severgnini et al. (in prep.).

(see Fig. 3, lower panels). A slightly less intense variation is also observed in the $2-10 \mathrm{keV}$ vs. $0.3-2 \mathrm{keV}$ ratio (about a factor of 2.4). During the new Swift monitoring the change in the spectral shape is less prominent, of a maximum factor of $\sim 1.5$ on timescales of $\sim 3.5-4$ days. No difference in the maximum amplitude of variation is observed when comparing the light curve of the $4-10 \mathrm{keV}$ vs. $0.3-4 \mathrm{keV}$ ratio with the light curve of the $2-10 \mathrm{keV}$ vs. $0.3-2 \mathrm{keV}$ ratio.

To summarise, the Swift monitoring allowed us to find Mrk 915 in a different state than previously detected, and to follow the source during a spectral variation. The origin of this behaviour is still under investigation; a detailed spectral analysis will be presented in Severgnini et al. (in prep.).

\section{Acknowledgments}

The research leading to these results has received funding from the European Commission Seventh Framework Programme (FP7/2007-2013) under grant agreement n. 267251 "Astronomy Fellowships in Italy" (AstroFIt). Support from the Italian Space Agency is acknowledged by LB (contract ASI INAF NuSTAR I/037/12/0). This work made use of data supplied by the UK Swift Science Data Centre at the University of Leicester. This research has made use of the Palermo BAT Catalogue and database operated at INAF - IASF Palermo. We also want to thank Neil Gehrels and the Swift Mission Operation Center to make every effort to get our ToO request scheduled.

\section{References}

[1] Antonucci 1993, Unified models for active galactic nuclei and quasars, ARA\&A, 31, 473

[2] G. Risaliti, M. Elvis, F. Nicastro, Ubiquitous Variability of X-Ray-absorbing Column Densities in Seyfert 2 Galaxies, ApJ $\mathbf{5 7 1}$ (2002) 234

[3] A.G. Markowitz, M. Krumpe, R. Nikutta, First X-ray-based statistical tests for clumpy-torus models: eclipse events from 230 years of monitoring of Seyfert AGN, MNRAS 439 (2014) 1403 
[4] D.N. Burrows et al., The Swift X-Ray Telescope, SSRv 120 (2005) 165

[5] W.H. Baumgartner et al., The 70 Month Swift-BAT All-sky Hard X-Ray Survey, ApJS 207 (2013) 19

[6] G. Cusumano et al., The Palermo Swift-BAT hard X-ray catalogue.III.Results after 54 months of sky survey, A\&A 524 (2010) A64

[7] S. Andreon et al., Scaling relations of the colour-detected cluster RzCS 052 at $z=1.016$ and some other high-redshift clusters, MNRAS 383 (2008) 102

[8] G. Risaliti et al., Rapid Compton-thick/Compton-thin Transitions in the Seyfert 2 Galaxy NGC1365, ApJL $\mathbf{6 2 3}$ (2005) 93

[9] G. Risaliti et al., Occultation Measurement of the Size of the X-Ray-emitting Region in the Active Galactic Nucleus of NGC1365, ApJL $\mathbf{6 5 9}$ (2007) 111

[10] R. Maiolino et al., “Comets” orbiting a black hole, A\&A 517 (2010) AA47

[11] V. Braito et al., NGC1365: A Low Column Density State Unveiling a Low Ionization Disk Wind, ApJ 796 (2014) 87

[12] G. Torricelli-Ciamponi et al., Search for X-ray occultations in active galactic nuclei, MNRAS 442 (2014) 2116

[13] B. Agís-González et al., Black hole spin and size of the X-ray-emitting region(s) in the Seyfert 1.5 galaxy ESO 362-G18, MNRAS $\mathbf{4 4 3}$ (2014) 2862

[14] J. Gofford, et al. The Suzaku view of highly ionized outflows in AGN - I. Statistical detection and global absorber properties, MNRAS $\mathbf{4 3 0}$ (2013) 60

[15] G. Miniutti et al., The properties of the clumpy torus and BLR in the polar-scattered Seyfert 1 galaxy ESO 323-G77 through X-ray absorption variability, MNRAS 437 (2014) 1776

[16] D. Grupe et al., XMM-Newton Observations of the Narrow-Line Seyfert 1 Galaxy Mrk335 in a Historical Low X-Ray Flux State, ApJ 681 (2008) 982

[17] D. Grupe et al., A Remarkable Long-term Light Curve and Deep, Low-state Spectroscopy: Swift and XMM-Newton Monitoring of the NLS1 Galaxy Mkn335, ApJS 199 (2012) 28

[18] A.L. Longinotti et al., The Rise of an Ionized Wind in the Narrow-line Seyfert 1 Galaxy Mrk335 Observed by XMM-Newton and HST, ApJ 766 (2013) 104

[19] T.J. Turner et al., X-Ray Characteristics of NGC3516: A View through the Complex Absorber, ApJ 733 (2011) 48

[20] E.M. Huerta et al., A Detailed Analysis of the High Resolution X-Ray Spectra of NGC3516: Variability of the Ionized Absorbers, ApJ 793 (2014) 61

[21] P. Uttley et al., Complex X-ray spectral behaviour of NGC4051 in the low flux state, MNRAS 347 (2004) 1345

[22] A.P. Lobban et al., Contemporaneous Chandra HETG and Suzaku X-ray observations of NGC4051, MNRAS 414 (2011) 1965

[23] K.A. Pounds et al., Fe K emission and absorption features in XMM-Newton spectra of Mrk766: evidence for reprocessing in flare ejecta, MNRAS 342 (2003) 1147

[24] L. Miller et al., The variable X-ray spectrum of Mrk766. I. Principal components analysis, A\&A 463(2007) 131

[25] T.J. Turner et al., The variable X-ray spectrum of Mrk766. II. Time-resolved spectroscopy, A\&A 475 (2007) 121

[26] G. Risaliti et al., X-ray absorption by broad-line region clouds in Mrk766, MNRAS 410 (2011) 1027

[27] R.C. Reis et al., X-Ray Spectral Variability in NGC3783, ApJ 745 (2012) 93

[28] N. Bennert et al., Size and properties of the narrow-line region in Seyfert 1 galaxies from spatially-resolved optical spectroscopy, A\&A 459 (2006) 55

[29] M.L. Trippe et al., A Multi-wavelength Study of the Nature of Type 1.8/1.9 Seyfert Galaxies, ApJ 725 (2010) 1749

[30] M.A. Malkan et al., A Hubble Space Telescope Imaging Survey of Nearby AGN, ApJS 117 (1998) 25 